\title{
EXAME FISICO: Opiniões e percepções de alunos de graduação em enfermagem
}

\author{
Denise de Andrade Dias Angelo ${ }^{12}$ \\ Carmen Maria Casquel Monti Juliani ${ }^{13}$ \\ Silvia Cristina Mangini Bocchi ${ }^{14}$
}

RESUMO: Este estudo visou conhecer as opiniões e percepções dos alunos de graduação em enfermagem acerca do exame físico no âmbito do conceito, dificuldades, utilização de informações médicas, assim como a visão do processo ensino-aprendizagem sobre a temática em questão. As autoras chegaram as seguintes conclusões: - os conceitos emitidos por eles demonstram a relevância do procedimento à assistência de enfermagem, divergindo quanto ao que avaliar; - o procedimento é considerado pelos mesmos como sempre necessário para a assistência de enfermagem, porém suas dificuldades estão relacionadas à falta de embasamento teórico-prático, despreparo dos docentes, tempo limitado nos estágios e falta de espaço físico adequado para realização do procedimento; - os conhecimentos teórico-práticos oferecidos pelos cursos de graduação não são suficientes para execução do exame físico; - manifestam insegurança na realização do exame físico, principalmente no procedimento de ausculta; - revelam que apesar do exame físico acarretar exposição ao paciente, o enfermeiro não deve deixar de realizá-lo em detrimento deste ser executado por outro profissional; dizem utilizar informações médicas para a execução do exame físico, porém este fato necessitaria de investigações para elucidar de quais dados estão servindo-se e porque os utilizam.

UNITERMOS: Exame físico - alunos de graduação em enfermagem opiniões - percepções.

\footnotetext{
${ }^{12}$ Professora Assistente do Curso de Graduação em Enfermagem da Faculdade de Medicina de Botucatu - UNESP e doutoranda na área de Enfermagem Fundamental na Escola de Enfermagem de Ribeirão Preto - USP.

${ }^{13}$ Professora Auxiliar de Ensino do Curso de Enfermagem da Faculdade de Medicina de Botucatu - UNESP e mestranda na área de Administração na Escola de Enfermagem da USP.

${ }^{14}$ Professora Assistente do Curso de Graduação em Enfermagem da Faculdade de Medicina de Botucatu - UNESP.
} 


\section{INTRODUÇÃO}

A preocupação com a formação do aluno de forma a atender as necessidades da população é a nossa busca constante no ensino da enfermagem. Daí, a proposta de estarmos avaliando o conhecimento e percepção dos mesmos, a respeito do exame físico de enfermagem.

Sentimos em nossa experiência assistencial e na docência, tratar-se de um assunto polêmico, já que o enfermeiro nem sempre sente-se totalmente seguro na realização do exame físico, o qual configura-se como base à Sistematização da Assistência de Enfermagem (S.A.E.).

A avaliação clínica, como parte integrante da assistência de enfermagem foi apresentada pela primeira vez por Maccain em 1965, visto que o exame físico, até então, era uma atividade restrita ao médico. ${ }^{5}$

No Brasil, a preocupação com a observação sistematizada na identificação dos problemas clínicos de enfermagem se iniciou em meados de 1968, quando Wanda de Aguiar Horta apresentou um roteiro de exame físico fundamentado no utilizado pela medicina, no entanto diferindo-se quanto aos seus objetivos. Posteriormente, a autora, operacionalizando seu modelo conceitual como método de trabalho norteado pelo método científico, propõe um processo de enfermagem, caracterizado como a "dinâmica das açōes sistematizadas e interrelacionadas, visando a assistência ao ser humano". Esse processo por sua vez, tem como primeiro passo o histórico de enfermagem composto pela entrevista e exame físico. ${ }^{10}$

O exame físico, proposto pela autora, caracteriza-se como um roteiro sumarizado para coleta de dados no sentido céfalo-caudal do paciente.

Outros estudos consideram o exame físico um momento de estabelecimento de confiança no relacionamento enfermeiro-paciente, no qual, caso o paciente apresente queixa e por sua vez a enfermeira explore-o com perícia, a credibilidade da profissional tende a aumentar aos olhos do paciente, pois o mesmo poderá observar que ela está levando a sério sua queixa. ${ }^{2}$

Ademais, o exame físico constitui-se da verificação dos sinais vitais e outras mensurações utilizando-se também das técnicas de inspeção, palpação, percussão e ausculta de todos os segmentos do corpo. ${ }^{14}$

Observamos que os autores se referem ao exame físico como parte integrante do processo de enfermagem, mostrando ser esse um consenso para a operacionalização da S.A.E. Dessa forma, constatamos na prática que é impossivel estabelecer um diagnóstico de enfermagem e elaborar prescrição e evolução consistentes, se não procedermos uma avaliação clínica adequada.

Salientamos que além do exame físico constituir-se como parte integrante da etapa de coleta de dados e de apoiar o estabelecimento de diagnósticos de enfermagem, é precedente ao processo decisório do enfermeiro, frente à assistência de enfermagem prestada por ele mesmo e pela sua equipe.

Como atividades regulamentadas pela Lei 7498/86, artigo 11 , a consulta de enfermagem e a prescrição de enfermagem são de competência privativa do enfermeiro. ${ }^{3}$ Sendo assim, somos do consenso que uma das variáveis necessárias para que o mesmo venha exercer aquilo que é de sua 
competência, torna-se necessário que ele domine questões teórico-práticas do exame físico.

Partindo do pressuposto de que o exame físico deva ser incorporado à prática sistematizada do exercício da enfermagem para se atingir a qualidade almejada e considerada que o uso profissional deste exame depende, não só da habilidade do enfermeiro na realização do procedimento, mas principalmente da capacidade de discarnimento para interpretação e análise dos significados dos dados levantados, decidimos realizar um estudo com graduandos em enfermagem, visando conhecer as opiniões e percepções dos mesmos acerca do assunto.

Assim sendo, priorizamos as seguintes variáveis: o conceito de exame físico, as dificuldades para efetuá-lo, as opiniões sobre a exposição do cliente, a utilização de informações médicas e a visão do processo ensinoaprendizagem na graduação. A razão de investigarmos tais variáveis adveio da experiência das autoras e de achados literários, os quais referenciam alguns fatores bloqueadores na realização do exame físico pelo enfermeiro de forma efetiva. Observamos que alguns autores referenciam que existe divergências quanto ao significado do termo exame físico entre os enfermeiros. ${ }^{7,8,12}$

\section{METODOLOGIA}

A população alvo constou de 169 estudantes regularmente matriculados no quarto ano de graduação em enfermagem de quatro universidades do interior $e$ capital do estado de São Paulo. Optamos pela escolha dos referidos alunos, pelo fato de estarem concluindo as disciplinas teórico-práticas profissionalizantes, portanto julgamo-os em melhores condições em manifestarem suas opiniões e percepções com relação ao exame físico.

Assim, a amostra compôs-se de 73 alunos, correspondendo a $43 \%$ da população alvo.

A intenção era abranger a população total, mas acabamos trabalhando com os alunos presentes nas universidades no dia da coleta de dados, em junho de 1993.

Realizamos a coleta de dados através de questionário elaborado especificamente para este estudo, contendo questões fechadas e abertas.

Os questionários foram aplicados nos alunos pelas próprias pesquisadoras, possibilitando aos mesmos o recebimento de orientações referentes ao objetivo do trabalho e sobre o preenchimento do impresso.

\section{RESULTADOS: APRESENTAÇÃO E DISCUSSÃO}

Os resultados serão apresentados descritivamente, seguidos de discussão conforme a seqüência do instrumento de coleta de dados.

Os conceitos de exame físico elaborados pelos alunos de quarto ano da graduação em enfermagem, demonstram a relevância do procedimento à assistência de enfermagem, no entanto, observou-se a existência de divergências entre eles quanto ao que se avaliar, principalmente, nas condições físicas, mentais, sociais e espirituais do paciente. 
Estudos ressaltam que o exame físico é uma atividade exercida durante o exame de enfermagem, quando se buscam dados fisiológicos e biológicos do cliente em questão ${ }^{12}$, apesar da palavra físico, de acordo com o dicionário, referir-se ao corpo distinto de mente ${ }^{8}$, o exame físico vem sendo caracterizado como "estudo psico-sócio-espiritual, através da observação, interrogatório, inspeção, testes e uso de instrumento".

O exame físico é considerado por $82 \%$ dos alunos como sempre necessário para a assistência de enfermagem $\in 18 \% \circ$ julgam às vezes necessário. Para aqueles que conjeturam como às vezes necessário, suas justificativas mais significativas para tal informação foram as seguintes:

"... depende do área de atuaçăo do enfermeiro assistencial ou ad ministratioo ...

As dificuldades quanto a realização do exame físico foram manifestadas por $73 \%$ dos alunos, das quais relacionadas principalmente: a falta de embasamento teórico-prático, o despreparo dos docentes, o tempo limitado nos estágios e a falta de espaço físico adequado.

Em se tratando da assistência de enfermagem, o enfermeiro é um profissional qualificado para desenvolver atividades que propiciem a identificação de problemas relacionados ao processo saúde-doença, podendo contribuir conjuntamente com a equipe multiprofissional, para a melhoria da assistência à saúde da população. ${ }^{9}$

Ressaltamos que para a obtenção de domínio e segurança na prática do exame físico, faz-se necessário embasamento teórico em anatomia, fisiologia, além de habilidades em técnicas básicas, como: inspeção, palpação, percussão e ausculta, as quais possuem características que as tornam difíceis de serem adquiridas sem orientação direta e específica.

Concordamos com estudos que enfatizam a necessidade dos enfermeiros se prepararem para a prática do exame físico. Essa competência requer que enfermeiros tanto na área de ensino quanto da assistência, conjuguem esforços no sentido de amenizar a falta de conhecimentos teórico-práticos com referência ao exame físico, estimulando o exercício de suas técnicas como atividades na assistência prestada a usuários dos serviços de saúde.

Quanto à avaliação do processo ensino-aprendizagem do exame físico oferecido pelos cursos de graduação em enfermagem, verificamos que $61 \%$ dos alunos referiram que eles não proporcionaram-lhes conhecimentos teóricopráticos suficientes para execução do mesmo.

Destacamos mais uma vez a responsabilidade do docente no processo ensino aprendizagem do exame físico, como um dos elementos responsáveis pela formação do aluno. Para tanto, faz-se necessário que o doente valorize e domine o conteúdo de maneira a não ser mero repassador de conhecimentos, mas sobretudo agente facilitador na reformulação de conceitos e de atitudes profissionais em prol de uma melhor qualidade assistencial. 
Dos investigados, $90 \%$ manifestaram insegurança na realização do exame físico, ordenando as técnicas por grau de maior para menor dificuldade, sendo ausculta, percussão, palpação e inspeção.

Muitas vezes a incapacidade em realizar algumas técnicas do exame físico (inspeção, palpação, percussão e ausculta), bem como a incompreensão quanto à riqueza de informação e uma gama de possibilidades que um exame físico completo pode oferecer, têm levado os enfermeiros a se restringirem a um exame simplificado e resumido. ${ }^{12}$

Com a preocupação de estudar outras questões que permeiam a realização do exame físico, evidenciamos que dos 73 alunos, $53 \%$ deles relataram não sentirem-se constrangidos ao expor o paciente durante o procedimento, já $44 \%$ referiram-se constrangidos e $1 \%$ não nos respondeu.

A exposição durante o exame físico, principalmente das genitálias, requer por parte do enfermeiro compreensão e respeito em relação às emoções do paciente. Recomenda-se que esse tipo de exame seja feito rapidamente, mas com rigor, passando a sensação ao paciente de estar sendo exposto apenas a área do órgão examinado, preservando ao máximo a sua individualidade.

Levantando a questão de que o exame físico constitui-se em atividades similares entre médicos e enfermeiros, acarretando excessiva exposição do paciente, $77 \%$ dos alunos pesquisados concordaram e $23 \%$ discordaram com essa proposição. Talvez, uma alternativa de solução para o problema seria a utilização de impresso comum, onde a necessidade de cada profissional se complementasse com vistas a finalidade única: 0 atendimento às necessidades do paciente.

Dos 73 alunos, $86 \%$ acham que o enfermeiro não deve deixar de realizar o exame físico, quando o mesmo já foi realizado por outro profissional, $10 \%$ acham que sim e $4 \%$ não nos respondeu.

Em se tratando da relação de papéis entre os profissionais da saúde, estudos da década de 70 afirmam que "tradicionalmente entende-se que o trabalho da enfermeira complementava o do médico", entretanto, atualmente "as relações entre os integrantes da equipe de enfermagem vêm tomando outro caráter, passando de uma situação de hierarquia a outra de corresponsabilidade", o que leva à conclusão de que o enfermeiro tem responsabilidades importantes com o doente, tanto quanto o médico; e ambos atuam no mesmo nível de autonomia, ao mesmo tempo em que um complementa o outro. ${ }^{12}$

Verificamos que $77 \%$ dos alunos nunca receberam reclamações por parte do paciente e/ou familiares, devido ao excesso de exposição causado pelo exame físico, já $23 \%$ receberam este tipo de queixa.

Ademais, dos 73 alunos pesquisados, $68 \%$ deles disseram utilizar-se de informações médicas para execução do exame físico, $26 \%$ relataram não servirem-se e $6 \%$ não nos respondeu.

A taxa elevada de alunos utilizando informações médicas para execução do exame físico, precisaria ser melhor investigada, para nos esclarecer de quais dados estão se servindo e porque os utilizam, para depois podermos inferir se 
essa atitude possa estar relacionada ou não à falta de conhecimentos teóricopráticos

Conforme podemos verificar no instrumento de coleta de dados, deixamos espaço para que o aluno fizesse alguma colocação que achasse oportuna, para tanto citamos algumas de suas sugestões

se almejamos uma assistência de enfermiagem de qualidade deoemos nos preparar na teoria e na prática pois somente assim terernos a credibilidade da pessoa assistida e de outros profissionais

quero lembrar que alérn de instrumento de coleta de dados, o exame fisico é um momento de interaçăo de enfermagem

deoeria existir uma reformulaçäo curricular, permitindo a criaçăo de um espaço. onde o exame físico terá uma atençăo especial e profunda

os professores deoeriam estar melbor preparados na teoria e prática. alérn de se padronizarem

o exame fúsico deoeria ser enfatizado por todas as disciplinas de forma uniforme

os estágios ... deoeriam ser melhores aprooeitados de forma que outras atioidades năo acarretern o prejuizo da execuçăo do exame físıco

\section{CONCLUSÕES}

A realização deste processo investigatório com alunos do quarto ano de graduação em enfermagem a respeito do exame físico, possibilitou-nos chegar às seguintes conclusões:

- os conceitos emitidos por eles demonstram a relevância do procedimento à assistência de enfermagem, divergindo quanto ao que avaliar no paciente;

- o procedimento é considerado pelos mesmos como sempre necessário para a assistência de enfermagem, porém suas dificuldades estão relacionadas à falta de embasamento teórico-prático, despreparo dos docentes, tempo limitado nos estágios e falta de espaço físico adequado para realização do procedimento;

- os conhecimentos teórico-práticos oferecidos pelos cursos de graduação não são suficientes para execução do exame físico;

- manifestaram insegurança na realização do exame físico, principalmente no procedimento de ausculta;

- apesar de acarretar exposição ao paciente, o enfermeiro não deve deixar de realizar o exame físico em detrimento deste ser realizado por outro profissional; 
revelam utilizar informações médicas para execução do exame físico, porém este fato necessitaria de investigações para elucidação de quais dados estão servindo-se e porque os utilizam.

É necessário que as instituições de ensino de enfermagem e de assistência comprometam-se com a reciclagem e o desenvolvimento profissional. Não há dúvida quanto à responsabilidade dos cursos de graduação no preparo dos profissionais que lançam ao mercado de trabalho, pois estes poderão tornar-se em agentes de mudanças dentro da realidade vivenciada. Enfermeiros assistenciais e docentes não podem furtar-se a dominar o conhecimento e os procedimentos que subsidiam a sistematização da assistência de enfermagem, visto que a mesma é uma atividade inerente à sua função. Para tal, o curso de graduação em enfermagem deverá representar o esteio do ensino formal, fundamentando os alunos para que num futuro próximo exerçam uma pratica assistencial cada vez mais eficaz.

Assim, acreditamos na importância da portaria 721/94/MEC que estabelece a inclusão da disciplina de semiologia e semiotécnica de enfermagem no currículo mínimo de graduação, não descartando também, a integração interdisciplinar na formação do enfermeiro, uma vez que, apenas a criação da referida disciplina não garantirá sua efetiva utilização. Contudo, precisamos que haja aplicação do exame físico como atividade diária dos enfermeiros.

ABSTRACT: This study aimed at getting to know the nursing graduate students' opnions and perceptions about concept of physical examination, difficulties, use of medical information, as well as, the vision of teaching-learning process about the theme in question. The authoresses concluded that: - the concepts expressed by them demonstrate the importance of behavior to the nursing assistance, diverging about to what evaluate; - the behavior is consider by themselves as always necessary for the nursing assistance, however, their difficulties are related to the lack of theorical-practical foundation, teachers not much prepared, limited time in the traineeships and lack of appropriate physical space for the executation of the behavior; - the theorical-pratical knowledge offered by the graduate courses aren't sufficient to the execution of the physical examinaton; - they show insecurity in the accomplishment of the physical examination, especially in the auscultation; - the students reveal that in spite of the physical exam result in exposing the patient, the nurse must not be want to achieve it to the detriment of this to be carried out by other profissionals; - it's said that they use medical information for execution of the physical examination, however, this fact would need inquiries to explain which datas they are using and why use them.

KEYWORDS: Physical Examination - Nursing Graduate Students Opnions - Perceptions. 


\section{REFERÊNCIAS BIBLIOGRÁFICAS}

1 - ADAMI, M. P. et al. Características básicas que diferenciam a consulta de enfermagem da consulta médica. Acta Paul. Enferm., São Paulo. v. 2, n. 1, p. 9-13, 1989.

2 - ATKINSON, L. D.; MURRAY, M. E. Fundamentos de enfermagem. Rio de Janeiro: Koogan, 1985. p. 215-31.

3 - BRASIL, Leis n. 7498/86, 26 jun. 1986. Dispõe sobre a regulamentação do exercício da enfermagem e dá outras providências. Diário Oficial da União, Brasília, 1986. Seção 1, p. 9273-5.

4 - BRASIL, Leis, etc. Portaria 721/94/MEC. Diário Oficial da União, Brasília, 16 dez. 1994. p. 1980-1.

5 - CAMPEDELLI, M. C. Processo de enfermagem. São Paulo: Ática, 1986. $136 \mathrm{p}$.

6 - CRANE, J. El exame físico: un aspecto en la evaluation clínica de enfermagem. In: SANA, J. M.; JUDGE, R. D. Métodos para el exame físico en la práctica de enfermaria. Cali: OPAS, 1977. p. 11-6.

7 - DANIEL, L. F. A enfermagem planejada. 3 ed., São Paulo: EPU, 1981. $33 \mathrm{p}$.

8 - FUERST, E. V. et al. Fundamentos de enfermagem. 4 ed., Rio de Janeiro: Interamericana, 1977. $491 \mathrm{p}$.

9 - GAMBA, M. A. "et al". Enfoque do exame físico sobre o prisma da saúde pública. In: Anais do ciclo de debates sobre sistematização do exame físico pelo enfermeiro, 1, São Paulo, 1990. Anais, São Paulo. p. 18.

10 - _. A opinião dos enfermeiros sobre o exame físico. Rio de Janeiro, 1990. Dissertação (Mestrado) - Universidade Federal do Espírito Santo.

11 - HORTA, W. A. Processo de enfermagem. São Paulo: EPU, 1979. 99p.

12 - OLIVEIRA, E. R. A. Proposta de um modelo de ensino do exame físico para o processo metodológico da assistência de enfermagem. Rio de Janeiro, 1990. 209 p. Tese (Livre-docência), Escola de Enfermagem Alfredo Pinto - Universidade Estadual do Rio de Janeiro.

13 - PAIM, L. Quantitativos e qualitativos do cuidado de enfermagem. Rio de Janeiro, 1977. Tese (Livre-docência), Escola de Enfermagem Ana Nery.

14 - POTTER, P. A.; PERRY, A. G. Fundamentals of nursing: concepts, process, and practice. 2 ed., St. Louis: Mosby, 1989. p. 117-8. 